P077 PATHOPHYSIOLOGICAL BASIS OF RESOLUTION OF ACUTE-ON-CHRONIC LIVER FAILURE (ACLF) INDUCED BY THE NOVEL LIVER DIALYSIS DEVICE, DIALIVE (ALIVER CONSORTIUM)

${ }^{1}$ Rafael Bañares Cañizares*, ${ }^{2}$ Faouzi Saliba, ${ }^{3,4}$ Dana Tomescu, ${ }^{5}$ Banwari Agarwal, ${ }^{6}$ Vanessa Stadlbauer, ${ }^{7}$ Gavin Wright, ${ }^{8}$ Mohammed Sheikh, ${ }^{9}$ Carrie Morgan, ${ }^{8}$ Fausto Andreola, ${ }^{10}$ Lennart Oettl, ${ }^{11}$ Carlos Alzola, ${ }^{12}$ Rahul Kumar, ${ }^{2}$ SophieCaroline Sacleux, ${ }^{6}$ Gernot Schilcher, ${ }^{9}$ Daniel Green, ${ }^{13}$ Sebastian Koball, ${ }^{5}$ Andrada Tudor, ${ }^{14}$ Jaak Minten, ${ }^{15} \mathrm{Gema}$ Domenech, ${ }^{15}$ Juan Jose Aragones, ${ }^{6}$ Karl Oettl, ${ }^{16} \mathrm{Katja}$ Waterstradt, ${ }^{17}$ Stefanie Bode-Boger, ${ }^{1}$ Maria-Vega Catalina, ${ }^{5} \mathrm{Amir}$ Gander, ${ }^{13,18} \mathrm{Jan}$ Stange, ${ }^{19}$ Moises Sanchez, ${ }^{8}$ Rajeshwar P Mookerjee, ${ }^{10}$ Andrew Davenport, ${ }^{8}$ Nathan Davies, ${ }^{20}$ Marco Pavesi, ${ }^{21}$ Javier Fernandez, ${ }^{13,18}$ Steffen Mitzner, ${ }^{20}$ Vicente Arroyo, ${ }^{8}$ Rajiv Jalan. ${ }^{1}$ Hospital Gregorio Maragnon, Madrid, Spain; ${ }^{2}$ AP-HP Hôpital Paul Brousse, Villejuif, France; ${ }^{3}$ Carol Davila University of Medicine and Pharmacy, Bucharest, Romania; ${ }^{4}$ Fundeni Clinical Institute, Bucharest, Romania; ${ }^{5}$ Royal Free Hospital, London, UK; ${ }^{6}$ Department of Internal Medicine, Division of Gastroenterology und Hepatology Medical University of Graz, Graz, Austria; ${ }^{7}$ Basildon and Thurrock University Hospital, Essex, UK; ${ }^{8} / L D H-U C L$, London, UK; ${ }^{9}$ Yaqrit, London, UK; ${ }^{10}$ UCL, London, UK; ${ }^{11}$ Eterasolutions, US; ${ }^{12}$ Changi General Hospital, Singapore; ${ }^{13}$ University Hospital Rostock, Rostock, Germany; ${ }^{14}$ Independent Clinical Research Organisation, Belgium; ${ }^{15}$ Medical Statistics Core Facility IDIBAPS - Hospital Clinic Barcelona, Barcelona, Spain; ${ }^{16}$ Medlnnovation GmbH, Germany; ${ }^{17 \prime}$ Institut für Klinische Pharmakologie Universitätsklinikum Otto-von-Guericke-Universität Leipziger, Germany; ${ }^{18}$ Fraunhofer IZI, Rostock, Germany; ${ }^{19}{ }_{1 B M}$, Ireland; ${ }^{20}$ EF-CLIF, Barcelona, Spain; ${ }^{21}$ Liver ICU, Liver Unit, Hospital Clinic Barcelona, Barcelona, Spain; ${ }^{22}$ Peninsula Medical School, University of Plymouth, Plymouth, UK

\subsection{6/gutjnl-2021-BASL.85}

Background In ACLF, systemic inflammation (SI) due to pathogen and damage associated molecular patterns (PAMPs and DAMPs), albumin and endothelial dysfunction results in organ failure. DIALIVE, a liver dialysis device replaces dysfunctional albumin and removes PAMPs and DAMPs. In a randomised controlled trial, DIALIVE resolved ACLF faster and in a greater proportion of patients compared with standard of care (SOC). This substudy aimed evaluated the effect of DIALIVE on pathophysiological processes that are causally associated with ACLF.

Methods Study included patients with ACLF 1-3a, randomized to DIALIVE or SOC. End points were evaluated at Day 10. DIALIVE group received treatments in the first 3-days (range 1-6). Plasma samples were obtained on Days 0, 5 and 10. A post-hoc inferential Mixed Models for Repeated Measurements analysis was performed to evaluate the statistically significant differences between groups at the Day 10. Changes in variables from baseline in the groups is shown in the figure 1 SAS system v.9.4 used.

Results 32 ACLF patients were randomised to DIALIVE ( $\mathrm{N}=17$; CLIF-OFs: 10.3 (1.6)) or SOC ( $\mathrm{n}=15$;CLIF-OFs: 9.9 (1.2)). 30-evaluable patients $(n=15$ each group) comprised this substudy. PAMPs: Endotoxin activity: The patient-level predefined reduction goals were 20\%. Marked advantage seen for DIALIVE (80\%) vs SOC (36.4\%) at Day 5. LAL assay. Significant reduction in DIALIVE group $(p=0.005)$. Albumin Function: DIALIVE increased human mercaptalbumin $(p<0.001)$ and reduced human nonmercaptalbumins 1 and $2(p=0.003$ and $\mathrm{p}=0.203)$; improved binding capacity $(\mathrm{p}=0.073$; EPR spectroscopy) and reduced ischemia modified albumin ratio $(\mathrm{p}=0.014)$ vs SOC. DAMPs: M30 and M65 components of cytokeratin $18 \quad(\mathrm{p}=0.079 ; \mathrm{p}=0.192)$ and RIPK3 (marker of necroptosis $)(p=0.624)$ were reduced in the DIALIVE group. SI: IL-8, IL-18 and TNFa were reduced in the DIALIVE compared with SOC group $(p=0.021 ; p=0.021 ; p=0.08)$. Endothelial function: ADMA and Factor VIII were markedly reduced in the DIALIVE patients $(p<0.001 ; p=0.070)$. TLR4 and Inflammasome ligands: Incubation of plasma with a 1L1b/ IL18 Inflammasome cell line reduced signalling significantly $(p=0.021)$; favourable reductions were also seen with TLR4 reporter cell line $(p=0.403)$, in favour of DIALIVE vs SOC.

Conclusions DIALIVE impacts significantly on DAMPs, PAMPS, albumin and endothelial function, markers of SI and, reduces TLR4 and inflammasome ligands that collectively contribute to its ability to resolve ACLF.

\section{P078 READMISSIONS POST LIVER TRANSPLANTATION: A 10 YEAR STUDY}

Thomas Manship*, Paul Brennan, Thomas Clouston, Sarah Dobson, Kenneth Simpson. NHS Lothian, Edinburgh, UK

\subsection{6/gutjint-2021-BASL.86}

Introduction Short-term readmissions post liver transplantation (LT) are a marker of patient care and a high-cost burden. Studies from the U.S. suggest a $30-45 \%$ readmission rate at
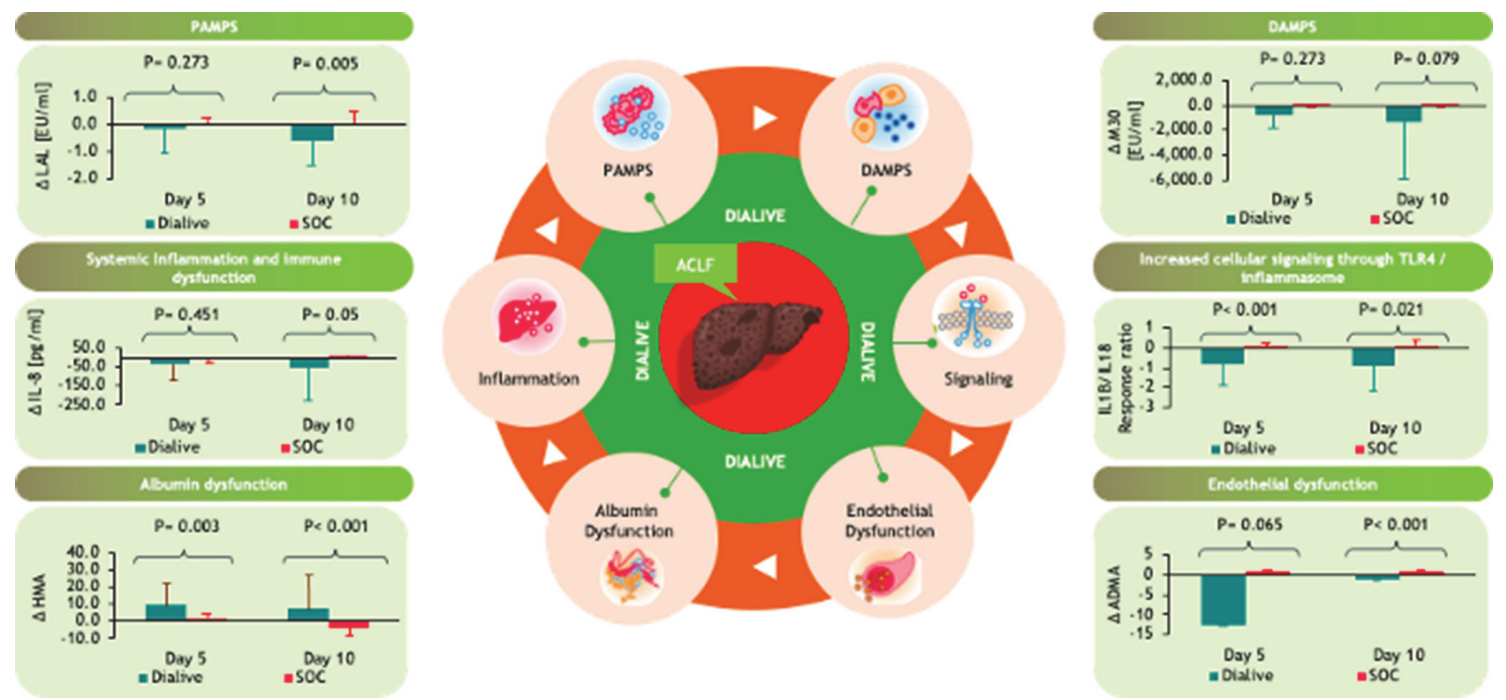

Abstract P077 Figure 1 Impact of DIALIVE on the pathophysiological processes deranged in ACLF - change from baseline at Days 5 and 10 for DIALIVE (green) and SOC (red) group 